\title{
Five shades of grey: Generalization in distractor-based retrieval of S-R episodes
}

\author{
Tarini Singh $^{1} \cdot$ Birte Moeller $^{1} \cdot$ Christian Frings ${ }^{1}$
}

Published online: 8 September 2016

(C) The Psychonomic Society, Inc. 2016

\begin{abstract}
Binding theories assume that a stimulus and a response made to it are bound together, as in the case of the theory of event coding, in an event file (Hommel, Müsseler, Aschersleben, \& Prinz, Behavioral and Brain Sciences, 24(05), 849-937, 2001). This binding occurs after even a single encounter with the stimulus and the response. Repeating any part of the event file will cause the entire file to be retrieved. However not only are relevant stimuli bound with responses but even irrelevant stimuli that co-occur with the target can be bound with the response, and repeating such a distractor will result in the event file being retrieved. Yet previous studies focused on retrieval effects due to repetition of the same distractor. In this experiment we analysed whether perceptually similar distractors still influence actions due to distractor-based retrieval of responses. Thirty-one participants responded to the shape of the stimulus while ignoring the luminance ( 5 different shades of grey). The similarity of the stimulus luminance between two consecutive trials influenced response times on response repetition trials. Response repetition effects were particularly facilitated with exact repetitions of the irrelevant feature luminance, and the strength of this effect diminished with increasing dissimilarity of luminance in a linear fashion. We conclude that response-retrieval effects due to distractor bindings follow the rules of generalization, as discussed in the conditioning literature.
\end{abstract}

Tarini Singh

singh@uni-trier.de

1 Cognitive Psychology Unit, University of Trier, D-54286 Trier, Germany
Keywords Perception and Action - Attention and executive control

Often, our actions in situations that require quick responses are fast and perceived as requiring relatively little effort. A number of processes allow the cognitive system to function in this manner. One these is the fast integration of stimulus (S) and response $(\mathrm{R})$ features into $\mathrm{S}-\mathrm{R}$ bindings, and the retrieval of such bindings (Henson, Eckstein, Waszack, Frings, \& Horner, 2014). S-R bindings are temporary associations between a stimulus and a response. If the stimulus is reencountered while the binding is still intact or can be retrieved, making the same response is faster because the required response information is available. Stimulus and response codes pertaining to an episode are integrated in an event file (Hommel, 1998, 2004; Hommel, Müsseler, Aschersleben, \& Prinz, 2001). An event file is thus a temporary structure containing bindings between stimulus and response feature codes of an episode.

Even irrelevant stimuli or features can be integrated into event files and are consequently integrated with the response- this has been called distractor-response binding (Frings, Rothermund, \& Wentura, 2007). Thus, repeating the distractor can retrieve the response with which it was integrated. After distractor-response integration, repeating the distractor or irrelevant feature is advantageous if the response is repeated because the retrieved response information matches the response to be made. By contrast, repetition of a distractor or irrelevant feature leads to a disadvantage if the response is changed because the retrieved response information is inappropriate. That is, distractor-response binding and retrieval is indicated by a significantly larger distractor repetition benefit in response repetition than in response change trials. Distractor-response binding effects are calculated as the 
difference of differences between distractor change and repetition in response repetition and change trials - that is, (RRDC-RRDR)-(RCDC-RCDR).

Considerable research using a prime-probe design with repeating or changing distractors (or irrelevant features) has reported evidence for distractor-based retrieval effects (e.g., Frings, 2011; Giesen \& Rothermund, 2014; Mayr \& Buchner, 2006; Rothermund, Wentura, \& De Houwer, 2005). Distractor-based retrieval of S-R episodes has been shown with different stimuli (e.g., words, colours, pictures; Frings et al., 2007; Frings, Moeller, \& Rothermund, 2013; Hommel, 1998; Hommel \& Colzato, 2009; Mayr \& Buchner, 2006; Moeller \& Frings, 2011; Moeller, Rothermund, \& Frings, 2012; Rothermund et al., 2005). However, in nearly all studies investigating distractorresponse binding effects, distractor repetition always implied a perceptually exact repetition of the distractor (see Frings et al., 2013, for an exception).

In a related paradigm, Denkinger and Koutstaal (2009) analysed S-R bindings between targets and responses if a perceptually and conceptually similar stimulus was repeated. They presented either the exact same item or a different exemplar of it (e.g., different pictures of a guitar). Still, Denkinger and Koutstaal observed evidence for retrieval of S-R episodes and concluded that such repetition effects can be generalized to new stimuli. In their study for perceptually similar stimuli, the concepts (e.g., guitar) of the presented objects were repeated and may have started response retrieval. However, because the extent of similarity between the original item and its different exemplars was not explicitly varied, it is difficult to decide what part of retrieval was due to concept repetition and what part due to repetition of perceptually similar stimulus features.

In this study we expand the idea of generalization of retrieval effects to distractor-based retrieval while keeping the contribution of distractor concept repetition constant. In addition, we systematically varied the degree of perceptual similarity between repeated irrelevant features. The theoretical motivation for systematically varying the degree of similarity between repeated irrelevant features stems from comparison of binding effects with learning (e.g., Colzato, Raffone, \& Hommel, 2006; Giesen \& Rothermund, 2014; Henson et al., 2014).

Although associations formed in learning paradigms are typically longer lasting than those in binding paradigms, there are similarities between binding and learning. For instance, Giesen and Rothermund (2014) discussed structural similarities between Pavlovian conditioning and distractor-response bindings. They compared the target in the distractor- response binding paradigm to the unconditioned stimulus in Pavlovian conditioning, the response to the target to the unconditioned response, and the distractor to the conditioned stimulus. Pairing the target (unconditioned stimulus) and the distractor (conditioned stimulus) leads to an association between target and distractor and distractor and response (see Giesen \& Rothermund, 2014, for a detailed discussion). If these structural similarities are more than a coincidence, and binding and conditioning indeed share similar mechanisms, then other phenomena observed for learning should be observable for binding effects. One example might be context-dependent retrieval (Godden \& Baddeley, 1975). Godden and Baddeley (1975) observed better performance when environmental context remained constant for learning and retrieval. Studies in S$\mathrm{R}$ bindings have observed a comparable effect. Repeating an item initially presented in a particular task context in a different context retrieved the initial task set (Waszak, Hommel, \& Allport, 2003). Thus, task set seems to be integrated in the event file and retrieved if the stimulus is repeated.

Here we focused on another possible example. Generalization is a central concept in Pavlovian conditioning: Conditioned responding can occur in circumstances that are similar to those in the learning stage but not exactly the same (Pearce, 1987). Closely related to generalization is the concept of generalization decrement: in circumstances similar but not exactly the same as in the learning stage, this transfer of learning is not complete. Thus, generalization is observed when at least some of the features from the training phase are repeated in the test phase and the strength of generalization is dependent upon how much is repeated or how similar the stimuli are (Pearce, 1987). Assuming binding and conditioning rely on comparable mechanisms, we hypothesized that generalization might also be observed in binding paradigms. In line with the principle of generalization decrement, the effect would be expected to decrease with increasing dissimilarity between the stimuli.

We used a prime-probe paradigm that orthogonally varied response and irrelevant feature repetition between prime and probe events (see Frings et al., 2007) and systematically varied perceptual deviations of the distractor feature between integration event and retrieval event. If binding mechanisms resemble mechanisms in Pavlovian conditioning, then we expected decreasing strength of the distractor-response binding effect with increasing perceptual differences.

\section{Experiment}

\section{Method}

Participants Thirty-one (5 male) students from the University of Trier participated for either partial course credit or monetary reimbursement. The median age of the participants was 21 years (range 18-26 years). All participants reported normal or corrected-to-normal vision. The sample size was calculated according to previous distractor-based binding effects, which typically lead to middle to large sized effects (Cohen's $d$ 
between .4 and 1). Thus, we planned to run $N=30$ participants, leading to a power of $1-\beta=.96$ (assuming an alpha $=$ .05) (GPower 3.1.9.2; Faul, Erdfelder, Lang, \& Buchner, 2007).

Design The experimental design consisted of two withinsubjects factors; response relation (repetition vs. change) and distractor relation (exact repetition vs. repetition with 1 step deviation vs. repetition with 2 steps deviation vs. repetition with 3 steps deviation vs. change).

Materials The experiment was run using E-Prime Software, Version 2.0. Four shapes (square, diamond, cross and triangle) and five lightness values $(10,30,50,70$ and 90 of the $L$ value in the LAB colour space; the $a$ and $b$ values were kept at zero) were varied orthogonally, resulting in 20 different stimuli. Stimuli were $1.43^{\circ} \times 1.43^{\circ}$. They were presented on a $3.34^{\circ}$ $\times 3.34^{\circ}$ black-and-white striped background. Stimuli were displayed on a standard LCD monitor with a white background.

Procedure Participants were tested individually in soundproofed chambers.

Experimental instructions were presented on-screen and summarized by the experimenter. Participants were asked to place their right hand index finger on the $J$ key and their left hand index finger of the $F$ key at the beginning of the experiment and were asked to respond to the shape of the stimulus with the appropriate key press. Two shapes were mapped to one key. Half the participants responded to the square and cross shapes with the $F$ key and the diamond and triangle shapes with the $J$ key. The other half received the opposite mapping.

The stimuli were presented in the middle of the screen. Each trial began with a fixation cross at the centre of the screen for 1,000 ms; participants were instructed to fixate this cross. The fixation cross was followed by the prime display, which remained on-screen until a response was detected, then a blank screen followed for $500 \mathrm{~ms}$, and then the probe display, which remained on screen until a response was detected. Once a response was detected, the next trial began. The experiment was divided into a practice and a test block. The practice block consisted of 32 trials, where participants learned the appropriate responses. Feedback was provided after every response, indicating whether it was correct or not. The test block consisted of 600 trials. After half of the trials, participants were instructed to take a self-paced break. In the test block, feedback was only provided after incorrect responses.

Responses were indicated by the form of the target. In response repetition (RR) trials, the form of the target in the prime and probe always indicated the same response. In response change (RC) trials, the form of the target in the prime and probe always indicated different responses. Lightness values were used as distractor features. In distractor repetition (DR) trials, lightness value of the stimulus could either be exactly repeated or could be repeated in lightness values of varying degrees of similarity (with similarity decreasing with increasing difference in lightness value). There were three different degrees of similarity, the most similar (one step deviation) varying by 20 units of the $L$ value, the middle by 40 (two steps deviation), and the least similar by 60 (three steps deviation). Trials including the largest possible lightness value change between prime and probe ( 80 units) were defined as distractor change (DC) trials (see Fig. 1).

\section{Results}

Reactions times Only trials with correct responses to both prime and probe were included in the analysis. Trials shorter than $200 \mathrm{~ms}$ or longer than 1.5 interquartile ranges above the third quartile of the RT distribution of the participant (Tukey, 1977) were not included in analysis. This resulted in a total of $11.73 \%$ of the data being excluded from the RT analysis $(0.01 \%$ fast responses, $4.78 \%$ slow responses, $3.2 \%$ prime error rate, and $3.74 \%$ probe error rate). See Table 1 for mean RTs and probe error rates.

In a 2 (target relation) $\times 2$ (response relation $) \times 5$ (distractor relation) MANOVA on probe response times, the factor target relation (repetition vs. change) did not further modulate any of the relevant effects. The same was true for probe error rates. Similar results have been reported in other studies (e.g. Giesen \& Rothermund, 2014). For the sake of clarity, we therefore collapsed the target-relation factor. Probe response times were analysed in a 2 (response relation) $\times 5$ (distractor relation) MANOVA with Pillai's trace as the criterion.

Significant main effects for response relation, $F(1,30)=$ $67.64, p<.001, \mathrm{p}^{2}=.69$, and distractor relation, $F(4,27)=$ $2.96, p=.038, \mathrm{p}^{2}=.31$ were observed indicating faster responses in trials with repeated responses and faster responses with increasing similarity of the distractors. The interaction of response relation and distractor relation was also significant, $F(4,27)=6.00, p=.001, \mathrm{p}^{2}=.47$, indicating that repeating the exact lightness value when the same response was required facilitated responding, and this facilitation weakened with increasing dissimilarity between lightness values. In the same sense, repeating the exact lightness value when the response changed from prime to probe interfered with responding, and this interference decreased with increasing dissimilarity between prime and probe lightness values. To pinpoint this interaction, distractor-response binding effects (i.e. the interaction of Response Repetition $\times$ Distractor Repetition) were computed and compared across different levels of lightness deviation. We used the largest possible deviation as the distractor change condition. Thus, four distractor-response binding effects could be computed —one with exact repetition of the distractor in the distractor repetition condition and three 

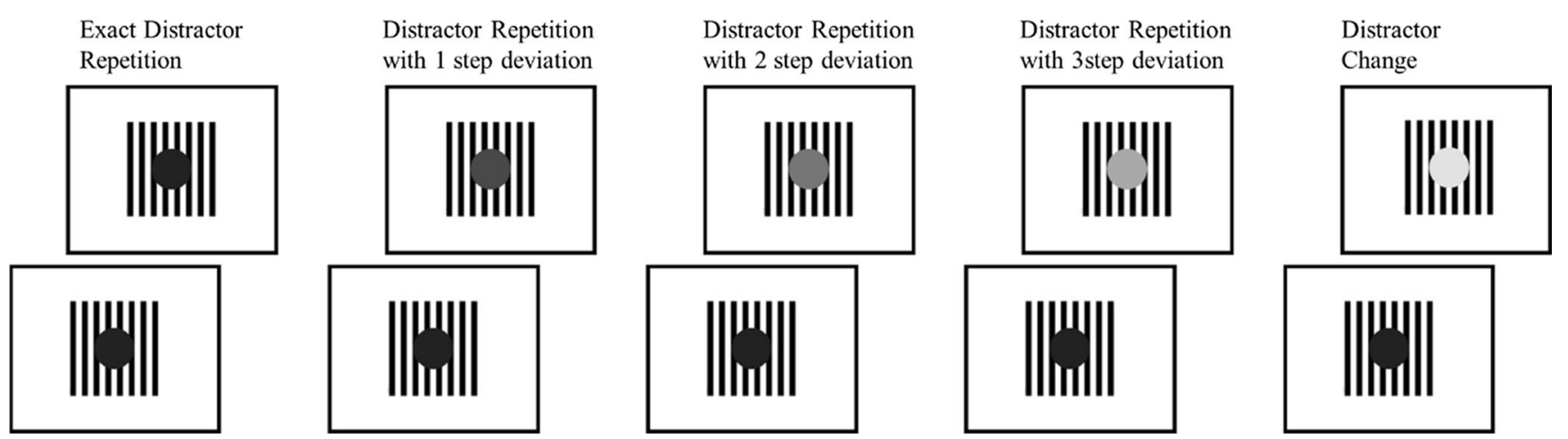

Fig. 1 Distractor repetition and distractor deviations. In exact repetition trials, the exact same lightness value was repeated. In distractor repetition trials with one step deviation, the next higher or lower lightness value was presented. In distractor repetition trials with two steps deviation, a lightness value that was two values higher or lower was presented. In

distractor-response binding effects with increasing lightness deviations in the distractor repetition condition. The distractor-response binding effects were submitted to a single-factor ANOVA, with the strength of deviation as the only factor. The strength of deviation was significant $F(3,28)$ $=5.81, p=.003, \mathrm{p}^{2}=.38$, indicating the largest distractorresponse binding effect for an exact replication and progressively weaker effects with increasing deviation. The linearly decreasing effects were reflected in the significant linear trend, $F(1,30)=11.29, p=.002,{ }_{\mathrm{p}}^{2}=.27$ (see Fig. 2a).

Error rates The same MANOVA was conducted on probe error rates yielding a significant interaction of response relation and distractor relation, $F(4,27)=3.7, p=.016, \mathrm{p}^{2}=.35$, indicating binding. None of the other effects were significant, $F \mathrm{~s}<3.7, p \mathrm{~s}>.229$. As with the RTs, the same four distractorresponse binding effects were computed and were submitted to a single-factor ANOVA, with the strength of deviation as the only factor. The strength of deviation was significant, $F(3$, 28) $=4.85, p=.008, \mathrm{p}^{2}=.34$, and the linearly decreasing effects were reflected in the significant linear trend, $F(1,30)=$ 15.48, $p<.001,{ }_{\mathrm{p}}^{2}=.34$ (see Fig. 2b).

Table 1 Mean reaction times (in $\mathrm{ms}$ ) and error rates in percentages (in parentheses)

\begin{tabular}{lll}
\hline & Response Repetition & Response Change \\
\hline $\begin{array}{lll}\text { Distractor Repetition } \\
\text { Exact Repetition }\end{array}$ & $479(2.95)$ & $525(5.60)$ \\
1 step deviation & $484(3.54)$ & $524(4.79)$ \\
2 steps deviation & $490(3.63)$ & $522(3.95)$ \\
3 steps deviation & $492(4.77)$ & $520(2.92)$ \\
Distractor Change & $495(3.91)$ & $524(4.51)$ \\
\hline
\end{tabular}

distractor repetition trials with three steps deviation, a lightness value that was three values higher or lower was presented. In distractor change trials, the lightness value that was four values higher or lower was presented

\section{Discussion}

The aim of the study was to test whether presentation of a similar but not exact same distractor could retrieve the response with which the distractor was bound, and whether the strength of this retrieval diminished with increasingly dissimilar distractors. Our results showed that this was indeed the case. Presentation of exactly the same distractor produced the strongest D-R binding effect. If the repeated distractor feature was similar but not exactly the same as the previously

\section{a Distractor-Response Binding effects}

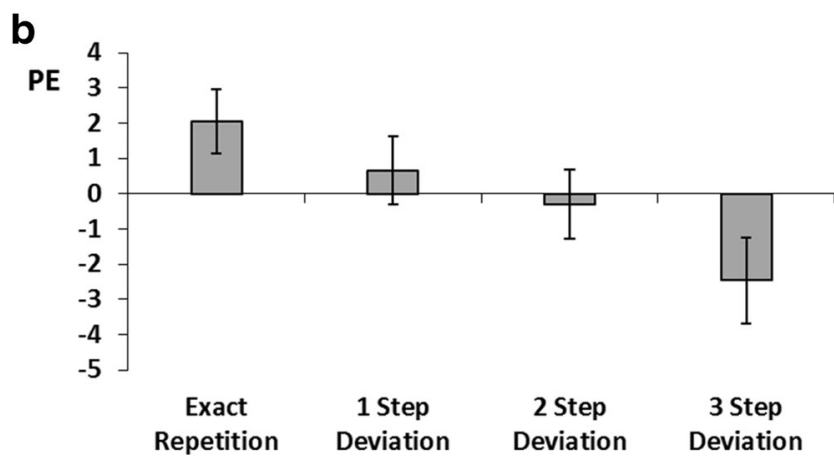

Fig. 2 Distractor-response binding effects in milliseconds for reaction times (a) and error percentages (b) for the four distractor repetition conditions. Distractor-response binding effects are calculated as the difference between distractor repetition effects in response repetition and response change trials

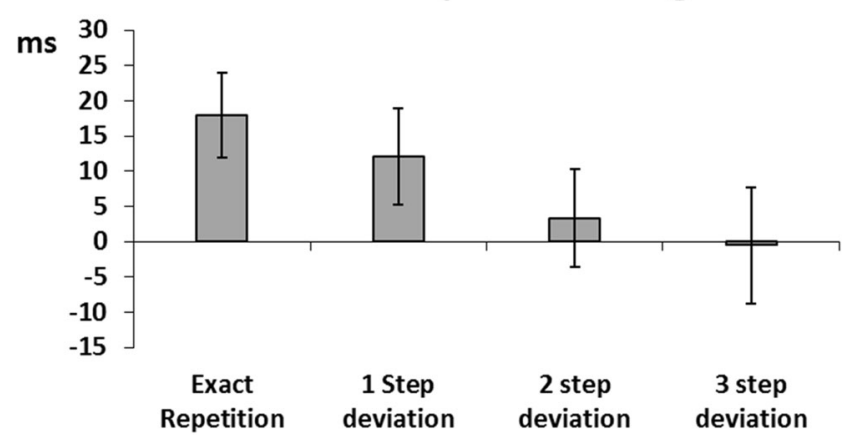


integrated one, we still observed binding effects. Yet the size of the binding effect decreased in a linear fashion as the dissimilarity between the distractor features in prime and probe increased.

This pattern of results is in line with the assumption that binding mechanisms are similar to mechanisms in Pavlovian conditioning. Applying the findings in conditioning to binding effects, the retrieval effect would indeed be expected to be strongest for exact repetitions but to generalize to perceptually similar stimuli. However, because the strength of generalization is dependent upon the similarity of the stimuli, the retrieval effect should also be stronger for stimuli that are similar than for stimuli with decreasing similarity. Apparently, after integrating a specific event file or instance (e.g. Logan, 1988), a stimulus showing large feature overlap with elements of the stored event file can trigger retrieval (Hommel, 1998; Moeller, Frings, \& Pfister, 2016). Moreover, these results indicate that the likelihood of retrieval also increases with increasing similarity of an individual retrieving feature. In a nutshell, these data suggest the generalization and generalization decrement processes observed in Pavlovian conditioning are also observed in the binding paradigm. This might be a further indication of similarity between Pavlovian conditioning and distractor-response binding — or learning and binding processes in general (see e.g. Giesen \& Rothermund, 2014; Henson et al., 2014; Moeller \& Frings, 2014).

This study was not the first to find response retrieval due to repetition of perceptually different distractors. Frings et al. (2013) used pictures and sounds of animals as distractors. Distractor repetition trials were realized via repetition of animal identity, while distractor modality could change (e.g., from a picture to a sound). They observed a retrieval effect, even if distractor modality switched between prime and probe. Notably, retrieval effects in the mentioned and the present studies are interpreted to rely on different mechanisms. Frings and colleagues (2013) presented evidence for response retrieval due to concept repetition, excluding retrieval due to repetition of perceptual features. Our results indicate larger retrieval effects with increasing perceptual similarity of integrated and retrieving distractor features.

Together these studies confirm the idea that S-R bindings are not just simple associations between a specific stimulus and specific response; rather, they appear to be structured bindings involving multiple levels of representation of responses, stimuli and tasks.

Our results are also in line with previous research in repetition priming. Priming effects are observed even with presentation of different exemplars of the same object. These effects, however, are not as strong as when the same exemplar is repeated (e.g. Biederman \& Cooper, 1991; more recently, Denkinger \& Koutstaal, 2009). The authors found priming effects for both exact repetition as well as repetition of a different exemplar of the object. However the priming effect for exact repetition was stronger than for repetition of different exemplars. Furthermore, they found that disruptions in priming due to decision changes occurred for both exact repetitions and repetitions of different exemplars. The authors argue that the bindings are "to some extent, abstract, in that they generalize to categorically and perceptually similar instances" (Denkinger \& Koutstaal, 2009, p. 750). In addition, the present results indicate retrieval generalization on a perceptual level is also possible without additional retrieval due to repetition of an object category.

Another theoretical approach that is often considered in connection with S-R binding is the instance theory of automatization (Logan, 1988). According to this theory, each encounter with a stimulus-response episode is encoded and stored as an instance in long-term memory. A repeated encounter with any stored stimulus retrieves all stored instances that include the stimulus, and this retrieval-based processing competes with the newly generated response. With an accumulation of instances, the probability increases that one of the retrieval processes wins this race. Hence, responding becomes memory based rather than algorithmic-processing based with increasing experience with a certain situation. It is difficult to distinguish instances and event files (e.g. Horner \& Henson, 2009; Moeller \& Frings, 2014). In fact, it has been speculated that bindings are individual steps in the learning process described by Logan (e.g. Logan, 1988; Moeller \& Frings, 2014). These finding seems to point to a crucial characteristic enabling bindings to contribute to learning. Because repetitions in a learning process are hardly ever exact, it appears to be adaptive that stimuli, sharing a large similarity with a stored stimulus, can also retrieve the response bound to this stimulus and further learning.

In conclusion, we found the principle of generalization, as suggested in conditioning research, also works in tasks that measure distractor-based retrieval of S-R episodes - retrieval of responses due to repetition of distractors or irrelevant features gradually declines with increasing dissimilarity of repeated distractors.

\section{References}

Biederman, I., \& Cooper, E. E. (1991). Evidence for complete translational and reflectional invariance in visual object priming. Perception, 20(5), 585-593.

Colzato, L. S., Raffone, A., \& Hommel, B. (2006). What do we learn from binding features? Evidence for multilevel feature integration. Journal of Experimental Psychology: Human Perception and Performance, 32(3), 705-716.

Denkinger, B., \& Koutstaal, W. (2009). Perceive-decide-act, perceivedecide-act: How abstract is repetition-related decision learning? Journal of Experimental Psychology: Learning, Memory, and Cognition, 35(3), 742-756. 
Faul, F., Erdfelder, E., Lang, A.-G., \& Buchner, A. (2007). G*Power 3: A flexible statistical power analysis program for the social, behavioral, and biomedical sciences. Behavior Research Methods, 39, 175-191.

Frings, C. (2011). On the decay of distractor-response episodes. Experimental Psychology, 58, 125-131.

Frings, C., Moeller, B., \& Rothermund, K. (2013). Retrieval of event files can be conceptually mediated. Attention, Perception, \& Psychophysics, 75(4), 700-709.

Frings, C., Rothermund, K., \& Wentura, D. (2007). Distractor repetitions retrieve previous responses to targets. The Quarterly Journal of Experimental Psychology, 60(10), 1367-1377.

Giesen, C., \& Rothermund, K. (2014). Distractor repetitions retrieve previous responses and previous targets: Experimental dissociations of distractor-response and distractor- target bindings. Journal of Experimental Psychology: Learning, Memory, and Cognition, 40(3), 645-659.

Godden, D. R., \& Baddeley, A. D. (1975). Context-dependent memory in two natural environments: On land and underwater. British Journal of Psychology 66, (3), 325-331.

Henson, R. N., Eckstein, D., Waszak, F., Frings, C., \& Horner, A. J. (2014). Stimulus- response bindings in priming. Trends in Cognitive Sciences, 18(7), 376-384.

Hommel, B. (1998). Event files: Evidence for automatic integration of stimulus-response episodes. Visual Cognition, 5(1/2), 183-216.

Hommel, B. (2004). Event files: Feature binding in and across perception and action. Trends in Cognitive Sciences, 8(11), 494-500.

Hommel, B., \& Colzato, L. S. (2009). When an object is more than a binding of its features: Evidence for two mechanisms of visual feature integration. Visual Cognition, 17(1/2), 120-140.

Hommel, B., Müsseler, J., Aschersleben, G., \& Prinz, W. (2001). Codes and their vicissitudes. Behavioral and Brain Sciences, 24(05), 849-937.

Horner, A. J., \& Henson, R. N. (2009). Bindings between stimuli and multiple response codes dominate long-lag repetition priming in speeded classification tasks. Journal of Experimental Psychology: Learning, Memory, and Cognition, 35(3), 757-779.

Logan, G. D. (1988). Toward an instance theory of automatization. Psychological Review, 95(4), 492.

Mayr, S., \& Buchner, A. (2006). Evidence for episodic retrieval of inadequate prime responses in auditory negative priming. Journal of Experimental Psychology: Human Perception and Performance, 32(4), 932-943.

Moeller, B., \& Frings, C. (2011). Remember the touch: Tactile distractors retrieve previous responses to targets. Experimental Brain Research, 214(1), 121-130.

Moeller, B., \& Frings, C. (2014). Long-term response-stimulus associations can influence distractor-response bindings. Advances in Cognitive Psychology, 10(2), 68-80.

Moeller, B., Frings, C., \& Pfister, R. (2016). The structure of distractorresponse bindings: Conditions for configural and elemental integration. Journal of Experimental Psychology: Human Perception and Performance, 42(4), 464-479.

Moeller, B., Rothermund, K., \& Frings, C. (2012). Integrating the irrelevant sound. Experimental Psychology, 59(5), 258-264.

Pearce, J. M. (1987). A model for stimulus generalization in Pavlovian conditioning. Psychological Review, 94(1), 61-73.

Rothermund, K., Wentura, D., \& De Houwer, J. (2005). Retrieval of incidental stimulus- response associations as a source of negative priming. Journal of Experimental Psychology: Learning, Memory, and Cognition, 31(3), 482-495.

Tukey, J. W. (1977). Exploratory data analysis. Reading, MA: AddisonWesley.

Waszak, F., Hommel, B., Allport, A. (2003). Task-switching and longterm priming: Role of episodic stimulus-task bindings in task-shift costs. Cognitive Psychology, 46, 361-413. 